\title{
Childhood intussusception: a multicentre study in a middle-income country
}

\author{
AE Ajao, ${ }^{1}$ FO Kumolalo, ${ }^{2}$ DI Olulana, ${ }^{3}$ OO Ogundoyin, ${ }^{3}$ TA Lawal ${ }^{3}$ \\ ${ }^{1}$ Department of Surgery, Bowen University Iwo and Bowen University Teaching Hospital, Nigeria \\ ${ }^{2}$ Department of Surgery, University of Medical Sciences and University of Medical Sciences Teaching Hospital Complex, Nigeria \\ ${ }^{3}$ Department of Surgery, University of Ibadan and University College Hospital, Nigeria
}

Corresponding author,email: akinlabi.ajao@gmail.com

Background: The outcome in infants and young children of intestinal obstruction due to intussusception is well documented in high-income countries. Our aim was to investigate the current pattern of presentation, management and outcome of childhood intussusception in a middle-income country using a multicentre approach.

Methods: Records of children managed for intussusception in three centres in South West Nigeria were retrospectively reviewed and analysed.

Results: One hundred and ninety children managed for intussusception were analysed. The male-to-female ratio was 1.8:1. The median age was 7 months with a peak age incidence of 3-6 months. Peak incidence was recorded during the dry season. Passage of bloody stool, vomiting, abdominal pain and fever were the most common presenting features. Seventy-three $(38.4 \%)$ of the patients presented with the classic triad. The median duration of symptoms was three days and only $19(10.1 \%)$ patients presented in less than 24 hours. Ileocolic intussusception was the most common anatomic type, occurring in $166(87.4 \%)$ patients, and was significantly associated with age. Twenty-two (56.4\%) had successful hydrostatic reduction, while $165(88.2 \%)$ required surgery. Forty $(21.1 \%)$ patients suffered postoperative complications. Eleven $(5.8 \%)$ of the patients died. The median duration of hospital stay was six days, and this correlated with the duration of symptoms and the length of hospital stay.

Conclusion: Delayed presentation is a persisting challenge in the management of childhood intussusception in Nigeria. Prompt referral to a paediatric surgeon may reduce the surgery rate, associated morbidity and the length of hospital stay. Keywords: childhood intussusception, delayed presentation, outcome, developing country

\section{Introduction}

Intussusception is the most common cause of intestinal obstruction in infants and young children. Peak incidence occurs between the ages of four and nine months, coinciding with the period of weaning from breast milk. ${ }^{1}$ It is commoner in males, in most reports, and shows some seasonal variations, with peaks coinciding with periods of acute viral infections. ${ }^{1-4}$ The aetiology is mainly idiopathic, but may also be secondary to pathologic lead points (PLPs). The patients usually present with any of the classic symptoms of abdominal pain or vomiting, or classic signs of abdominal mass or rectal bleeding. 5 Ileocolic intussusception is the most common type, followed by the ileoileocolic type. ${ }^{1,5,6}$ Diagnosis is mainly clinical and radiological. Ultrasound is a readily available and accurate modality for diagnosis and is usually very reliable..$^{5,6}$

The management of this condition is now mainly nonoperative in developed countries using pneumatic or hydrostatic reduction. ${ }^{6}$ However, in most low- and middleincome countries (LMICs), surgery remains the mainstay of treatment due partly to delayed presentation and late referrals of patients. . $^{3,7-10}$ The bowel resection rate is also high with consequent high morbidity and mortality. ${ }^{3,7,10}$ This is in contrast to reports from high-income countries. ${ }^{11}$ Low levels of literacy and limited access to specialist paediatric surgical care in LMICs may account for these adverse outcomes.

Following previously reported poor outcomes of management of childhood intussusception, there is the need for an audit to assess improvement in practice over time. This study hoped to use a multicentre approach to seek a broader perspective of childhood intussusception in Nigeria. The objective of this study was, therefore, to determine the presentation, management and outcome of childhood intussusception in a middle-income country using a multicentre approach.

\section{Methods}

Ethical approval was obtained for the conduct of this study. Children less than 15 years managed for intussusception between 2006 and 2017 at the University College Hospital (UCH), Ibadan, University of Medical Sciences Teaching Hospital Complex (UMSTHC), Ondo and Bowen University Teaching Hospital (BUTH), Ogbomoso, Nigeria, were reviewed.

$\mathrm{UCH}$ is a federal government-owned tertiary institution. UMSTHC is a state government-owned tertiary health centre, while BUTH is a private-owned tertiary hospital. The three hospitals, representing the three different types of tertiary hospitals with specialist paediatric surgery services 
in the region, were selected for a mix of patients from various socio-demographic groups.

\section{Statistics}

Data were collected on patients' demographics, pattern of presentation, management and outcome of treatment. Categorical variables were presented as frequencies, proportions and ratios, while continuous variables were presented as median and ranges. Associations between categorical variables were tested using the chi square or Fisher's exact test statistic. Correlation between continuous variables was tested using Spearman's rank correlation. Comparison of continuous variables across categorical groups was done using the Mann-Whitney U or the KruskalWallis tests. All data analyses were done using SPSS version 23.0 (Armonk, NY: IBM Corp)

\section{Results}

One hundred and ninety-four patients were managed for intussusception in the three institutions during the study period. Four of these were excluded because of their missing medical records. Figure 1 shows the distribution of the patients by the managing hospital, the majority $(153,80.5 \%)$ of which were seen at UCH. One hundred and twentytwo $(64.2 \%)$ were males and $68(35.8 \%)$ females, giving a male-to-female ratio of $1.8: 1$. The male-to-female ratio increased from 1.7:1 among infants to $2.2: 1$ in those older than 12 months. The median age was seven months with a range of one month to nine years. The peak age incidence was 3-6 months with a modal age of five months (Table I). One hundred and sixty-one $(84.7 \%)$ were infants, while only seven $(3.7 \%)$ were older than three years.

Peak incidence of intussusception was observed in January, February, June and October (Figure 2). The majority (125, $65.8 \%$ ) occurred during the dry season months of October to April. There was no significant difference in the proportion of those with preceding coryza during the dry or wet seasons $(p=0.339)$. Passage of bloody stool, vomiting, abdominal pain and fever were the most common features (Table II). Infants were more likely to present with vomiting and fever $(p=0.033)$, Table III. Seventy-three $(38.4 \%)$ patients presented with the classic triad of abdominal pain, abdominal mass and rectal bleeding. Twelve $(6.3 \%)$ patients presented with complications, including seizures (10), aspiration pneumonitis (1) and acute kidney injury (1). The median duration of symptoms was three days (range: 2 hours to 28 days). Nineteen (10.1\%) patients presented in less than 24 hours, while most $(46.6 \%)$ presented between $1-3$ days of onset of symptoms (Table II).

Clinical diagnosis was complemented by ultrasonography in $134(70.5 \%)$; plain radiography in $6(3.2 \%)$; and both in $10(5.3 \%)$ patients. In three $(1.6 \%)$ patients, ultrasonography was false-negative, giving an ultrasound sensitivity of 98.0\% (144/147). Hydrostatic reduction was attempted in 39 patients and successful in $22(56.4 \%)$ patients. However, one of those with successful hydrostatic reduction suffered a colonic perforation requiring surgical repair. Eight (4.2\%) patients had spontaneous reduction identified at surgery, evidenced by hyperaemia and oedema of the caecum and terminal ileum. Manual reduction was performed in 55 $(29.4 \%)$ and $102(54.5 \%)$ required bowel resection for gangrene $(78.4 \%)$, irreducibility $(12.4 \%)$, PLPs $(8.2 \%)$ and intestinal perforation $(1.0 \%)$. Three patients were taken away against medical advice giving an overall surgery rate of $88.2 \%$ (165 of 187). Ileocolic intussusception (166, $87.4 \%$ ) was the most common anatomic type (Table IV). Ninety per cent of ileocolic intussusception occurred in

\section{Number of patients}

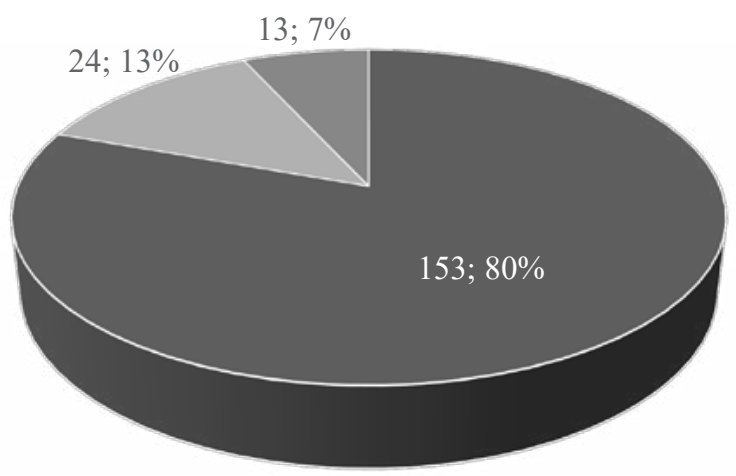

\section{UCH UMSTHC $\square$ BUTH}

Figure 1: Distribution of patients managed for intussusception by health institution

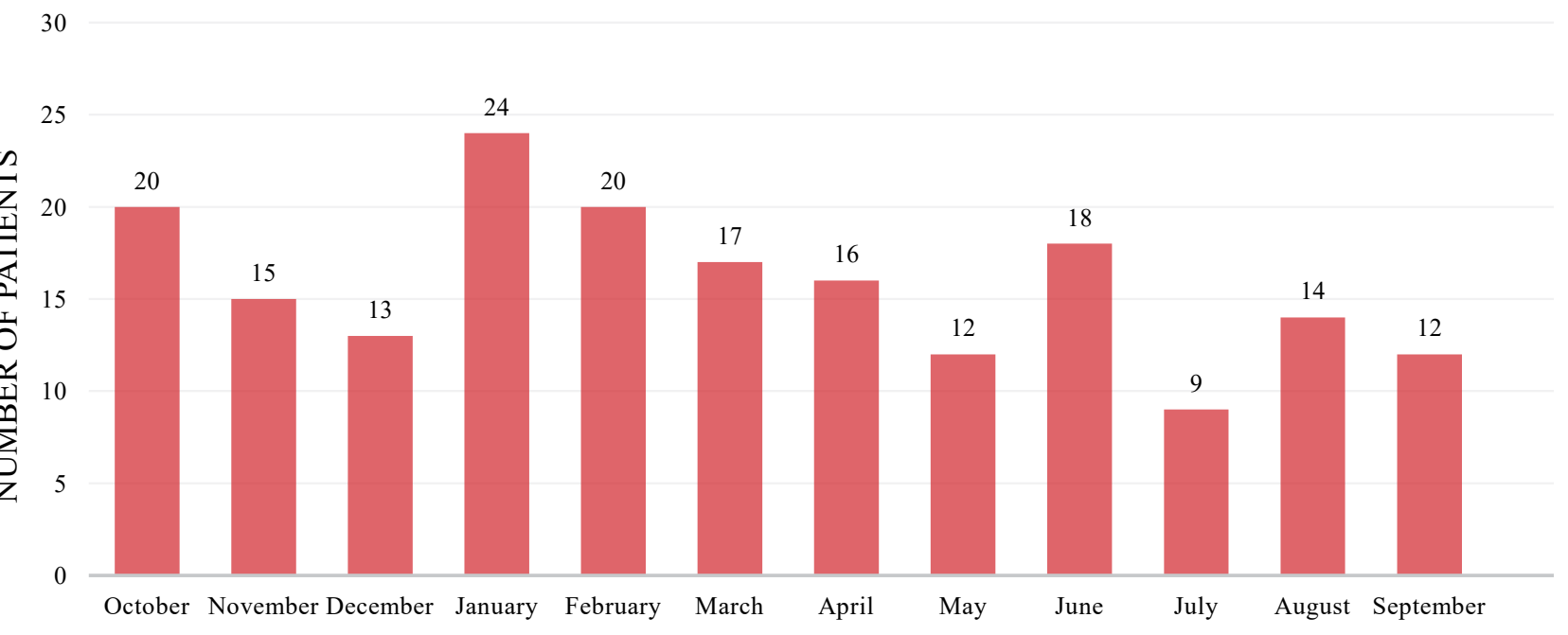

MONTH OF PRESENTATION

Figure 2: Monthly incidence of intussusception 


\begin{tabular}{|c|c|c|c|}
\hline Age group & Male (\%) & Female (\%) & Total (\%)* \\
\hline$<3$ months & $1(100.0)$ & $0(0.0)$ & $1(0.5)$ \\
\hline $3-6$ months & $51(58.0)$ & $37(42.0)$ & $88(46.3)$ \\
\hline $7-9$ months & $37(69.8)$ & $16(30.2)$ & $53(27.9)$ \\
\hline $10-12$ months & $13(68.4)$ & $6(31.6)$ & $19(10.0)$ \\
\hline$>12$ months -3 years & $14(63.6)$ & $8(36.4)$ & $22(11.6)$ \\
\hline$>3$ years & $6(85.7)$ & $1(14.3)$ & $7(3.7)$ \\
\hline Total & $122(64.2)$ & $68(35.8)$ & $190(100.0)$ \\
\hline
\end{tabular}

*Column percentages reported

\begin{tabular}{lll}
$\begin{array}{l}\text { Table II: Distribution of clinical features and time to } \\
\text { presentation among the patients }\end{array}$ \\
\hline Clinical presentation & Frequency, $\boldsymbol{n}$ & Percentage \\
\hline Features $(\boldsymbol{n}=\mathbf{1 9 0})$ & & \\
Bloody stool & 178 & 93.7 \\
Vomiting & 174 & 91.6 \\
Abdominal pain & 136 & 71.6 \\
Fever & 136 & 71.6 \\
Dehydration & 122 & 64.2 \\
Abdominal mass & 97 & 51.1 \\
Abdominal distension & 97 & 51.1 \\
Pallor & 71 & 37.4 \\
Dyspnoea & 47 & 24.7 \\
Palpable rectal mass & 42 & 22.1 \\
Preceding acute coryza & 33 & 23.2 \\
Recent diarrhoea & 19 & 10.0 \\
Anal protrusion & 14 & 7.4 \\
Complications & 12 & 6.3 \\
\hline Duration of symptoms in days $(\boldsymbol{n}=\mathbf{1 8 9})$ & \\
$<1$ & 19 & 10.1 \\
1-3 & 88 & 46.6 \\
4-6 & 59 & 31.2 \\
$\geq 7$ & 23 & 12.1 \\
& & \\
\hline
\end{tabular}

infants $(p=0.001)$, while the colocolic type was exclusive to older children. Polyps and Merkel diverticulum were found as PLPs in eight (4.8\%) of the 165 patients who had laparotomy.

Forty $(21.1 \%)$ patients had postoperative complications, with wound infection $(19,10.0 \%)$ predominating. Other complications included: wound dehiscence $(8,4.2 \%)$, severe sepsis $(4,2.1 \%)$, recurrent intussusception $(3,1.6 \%)$,

enterocutaneous fistula $(2,1.1 \%)$, and one each of bowel perforation post-hydrostatic reduction, adhesive intestinal obstruction and acute kidney injury. Occurrence of a complication was significantly associated with the presence of abdominal mass $(p=0.038)$ and anaemia $(p=0.010)$ at presentation but not with age $(p=0.298)$ or duration of symptoms $(p=0.139)$.

The median duration of hospital stay was six days with a range of one to 32 days. There was a weak but statistically significant positive correlation between the duration of symptoms and the length of hospital stay $(r=0.23, p=$ $0.002, n=190)$. The length of hospital stay was associated with the mode of treatment $(p<0.0001)$ and occurrence of complications $(p<0.0001)$. Eleven $(5.8 \%)$ of the patients died.

\section{Discussion}

This study summarises the presentation and management of childhood intussusception in three major health institutions in South West Nigeria, giving a broad overview in fairly different settings across the sub-region. In infants and toddlers, intussusception is the most frequent cause of intestinal obstruction. ${ }^{1}$ In developing countries, where surgery remains the mainstay of treatment, the implication is that of a significant surgical burden among children.

Eighty-five per cent of patients in this study were infants in keeping with reports of $75-89 \%$ from other parts of the country. ${ }^{3,12}$ The median age at presentation was five months and $46 \%$ of cases were between three and six months of age. Although higher peak age incidence of 6-9 months has been reported, ${ }^{3,13}$ this slightly lower peak was also observed by Bode in Lagos, Nigeria. ${ }^{7}$ This may reflect the earlier age at which children are weaned nowadays as the number of working-class mothers in society is increasing. Maternity leave in Nigeria still remains limited to only three months post-partum. This study also shows a male predilection as seen in most series, which was more evident in those older

Table III: Association of clinical characteristics with age

\begin{tabular}{lcccc}
\hline Variable & \multicolumn{2}{c}{ Age group } & Statistic & \multirow{p}{c}{-value } \\
\cline { 2 - 4 } & Infants & Older children & & $\mathbf{0 . 0 2 4 * \dagger}$ \\
\hline Vomiting & $150(95.5 \%)$ & $24(82.8 \%)$ & 0.16 & 0.692 \\
Abdominal pain & $114(78.1 \%)$ & $22(81.5 \%)$ & & $0.398 \dagger$ \\
Bloody stool & $152(94.4 \%)$ & $26(89.7 \%)$ & 0.50 & 0.481 \\
Abdominal mass & $81(51.9 \%)$ & $16(59.3 \%)$ & 4.54 & $\mathbf{0 . 0 3 3 *}$ \\
Fever & $120(76.4 \%)$ & $16(57.1)$ & 11.44 & $\mathbf{0 . 0 0 1 *}$ \\
Ileocolic intussusception & $122(75.8 \%)$ & $13(44.8 \%)$ & &
\end{tabular}

Percentages shown are within age groups; $\uparrow$ Fisher's exact statistic reported; ${ }^{*} p<0.05$ 


\begin{tabular}{lcc}
$\begin{array}{l}\text { Table IV: Distribution of the type of intussusception and } \\
\text { management instituted }\end{array}$ & Frequency, $\boldsymbol{n}$ & Percentage \\
\hline Variable & & \\
\hline Type of intussusception & 166 & 87.4 \\
Ileocolic & 9 & 4.7 \\
Colocolic & 5 & 2.6 \\
Ileoileocolic & 4 & 2.1 \\
Ileoileal & 4 & 2.1 \\
Ileocaecocolic & 1 & 0.5 \\
Jejunoileal & 1 & 0.5 \\
Caecocolic & & \\
\hline Treatment ( $\boldsymbol{n}=\mathbf{1 8 7 )}$ & 21 & 11.2 \\
Hydrostatic reduction & 55 & 29.4 \\
Surgery and manual reduction & 102 & 54.5 \\
Surgery and resection & 8 & 4.2 \\
Spontaneous reduction & 1 & 0.5 \\
Surgery and repair of & & \\
post-hydrostatic reduction & &
\end{tabular}

than a year. The reason for this sex difference remains unclear.

Intussusception has been observed to display seasonal variations that coincides with an increased frequency of adeno and rotavirus infections. ${ }^{5,6}$ In Nigeria and Tanzania, an increased incidence has been observed in the dry season. ${ }^{3,7,8,12}$ In the present study, peak incidences were observed in January, February, June and October (Figure 2). Sixty-six per cent of cases occurred during the dry season. This was in contrast to an Ethiopian report by Belachew et al. that documented an increased occurrence during the wet season peaking in June. ${ }^{14}$ Seasonal variation was, however, not observed in a report from Austria. ${ }^{15}$

The most common symptoms were bloody stools (94\%) and vomiting (92\%) in contrast to the observation of abdominal pain $(98 \%)$ from some developed countries. ${ }^{2}$ Bloody stools, being a late event in the pathogenesis of the disease, suggests late presentation in these patients. Vomiting and fever were more prominent in infants compared to older children (Table III). Acute onset of bilious vomiting and fever in an otherwise healthy infant should therefore raise the suspicion of intussusception. The classic triad of abdominal pain, abdominal mass and passage of currant jelly stool was observed in $38 \%$ of the patients, similar to findings of $33 \%$ in Jos and Enugu in Nigeria. ${ }^{9,12}$ Although, higher rates of $42-61 \%$ have been reported, ${ }^{2,7,8}$ the triad may not be reliable in excluding intussusception.

The median duration of symptoms was three days, with only $10 \%$ of the patients presenting within the first 24 hours of observed symptoms. This is in contrast to the $25 \%$ earlier reported from UCH, Ibadan between 2002 and 2011. ${ }^{10}$ Only $7-15 \%$ of patients present within the first day in reports from Africa and Asia. ${ }^{3,16,17}$ These are in contrast to the $74 \%$ reported by Lehnert et al. in Germany and other high-income countries. ${ }^{2}$ Delayed presentation has been shown to be associated with high bowel resection and mortality rates. ${ }^{10,16}$

Ultrasonography and plain abdominal radiography were used to complement clinical diagnosis. The diagnostic accuracy of ultrasound was $98 \%$ in the present study, in keeping with $98-100 \%$ sensitivity reported by Daneman et al. as cited by Lehnert et al. ${ }^{2}$ Ileocolic intussusception was the most common anatomic type and was significantly more common in infants. Colocolic intussusception was the next most frequent type, similar to findings in Enugu, Nigeria. ${ }^{9}$ PLPs were present in five per cent of patients, similar to the $2-9 \%$ reported in the literature. ${ }^{2,3,8,9}$ A higher PLP rate of $21.2 \%$ was observed in a Nepal study. ${ }^{17}$ However, the mean age of the cohort in the latter study was 46 months with most patients presenting after the age of four years, suggesting that PLPs are predominant in older children.

Surgery is the mainstay of treatment in this environment, accounting for $88.2 \%$ of cases in this series. Non-operative reduction was carried out in only one of the centres (UCH), while the other centres lacked the logistics. The bowel resection rate was $55 \%$, similar to other findings around the country. ${ }^{3,4,7,12}$

Complication rate in this study was $21 \%$. A palpable abdominal mass and observation of pallor at presentation were significantly associated with the eventual development of complication following treatment. The mortality rate of $5.8 \%$ reported in this series is a marked improvement from the $12-15 \%$ reported a few years back in this environment. $3,7,8$ The median duration of admission in the hospital following treatment was six days. Previous studies had reported mean lengths of stay of 7-17 days, which are higher than the average observed in this study.,7-9,17 This may have been due to the slightly higher surgical and complication rates in those studies. The length of hospital stay was significantly affected by the duration of symptoms at presentation, mode of treatment and the development of postoperative complications. Patients who had operative treatment and bowel resection were particularly more likely to have prolonged stay as earlier reported..$^{8,11}$

\section{Conclusion}

Delayed presentation is a persisting challenge in the management of childhood intussusception in developing countries. Presentation of an infant with fever, vomiting and abdominal pain during the dry season should raise the physician's index of suspicion. Prompt referral to a paediatric surgeon may reduce the surgery rate, associated morbidity and the length of hospital stay.

\section{Conflict of interest}

The authors have no conflict of interest to declare.

\section{REFERENCES}

1. Maki AC, Fallat ME. Intussusception. In: Ashcraft KW, Holcomb GW, Murphy JP, Ostlie DJ, editors. Ashcraft's Pediatric Surgery. London; New York: Saunders/Elsevier; 2014. p. 531-8.

2. Lehnert T, Sorge I, Till H, Rolle U. Intussusception in children - clinical presentation, diagnosis and management. Int J Colorectal Dis. 2009;24(10):1187-92.

3. Talabi AO, Sowande OA, Etonyeaku CA, Adejuyigbe O. Childhood intussusception in Ile-Ife: What has changed? Afr J Paediatr Surg. 2013;10(3):239-42.

4. Ogundoyin OO, Olulana DI, Lawal TA. Childhood intussusception: A prospective study of management trend in a developing country. Afr J Paediatr Surg. 2015;12(4):217-20. 
5. Columbani PM, Scholz S. Intussusception. In: Coran AG, Adzick NS, editors. Pediatric surgery. Philadelphia, PA: Elsevier/Saunders; 2012. p. 1093-110.

6. Marsicovetere P, Ivatury SJ, White B, Holubar SD. Intestinal Intussusception: Etiology, Diagnosis, and Treatment. Clin Colon Rectal Surg. 2017;30(1):30-9.

7. Bode CO. Presentation and management outcome of childhood intussusception in Lagos: a prospective study. Afr J Paediatr Surg. 2008;5(1):24-8.

8. Chalya PL, Kayange NM, Chandika AB. Childhood intussusceptions at a tertiary care hospital in northwestern Tanzania: a diagnostic and therapeutic challenge in resourcelimited setting. Ital J Pediatr. 2014;40(1):28.

9. Ekenze SO, Mgbor SO, Okwesili OR. Routine surgical intervention for childhood intussusception in a developing country. Ann Afr Med. 2010;9(1):27-30.

10. Ogundoyin OO, Olulana DI, Lawal TA. Childhood intussusception: Impact of delay in presentation in a developing country. Afr J Paediatr Surg. 2016;13(4):166-9.

11. Somme S, To T, Langer JC. Factors determining the need for operative reduction in children with intussusception: a population-based study. J Pediatr Surg. 2006;41(5):1014-9.
12. Ugwu BT, Legbo JN, Dakum NK, et al. Childhood intussusception: a 9-year review. Ann Trop Paediatr. 2000;20(2):131-5.

13. Palupi-Baroto R, Lee KJ, Carlin JB, Bines JE. Intussusception in Australia: epidemiology prior to the introduction of rotavirus vaccine. Aust N Z J Public Health. 2015;39(1):11-4.

14. Belachew AG, Tadesse A, Bogale BH. Patterns and seasonal variation of intussusception in children: a retrospective analysis of cases operated in a tertiary hospital in Ethiopia. Ethiop Med J. 2016;54(1):9-15.

15. Saxena AK, Höllwarth ME. Factors influencing management and comparison of outcomes in paediatric intussusceptions. Acta Paediatr. 2007;96(8):1199-202.

16. Ekenze SO, Mgbor SO. Childhood intussusception: the implications of delayed presentation. Afr J Paediatr Surg. 2011;8(1):15-8.

17. Shakya VC, Agrawal CS, Sinha AK, et al. Childhood intussusception: a prospective institutional study at BPKIHS. J Nepal Paediatr Soc. 2011;31(1):6-10. 